\title{
Secondary Osteosarcoma After Bone Marrow Transplant: An Aggressive Disease
}

\author{
Marta Pierobon, MD, ${ }^{1}$ Federico Mercolini, MD, ${ }^{2}$ Maria Carmen Affinita, MD, ${ }^{1}$ \\ Lucia Tombolan, BSc, ${ }^{3}$ Laura Battisti, MD, ${ }^{2}$ and Gianni Bisogno, MD, PhD ${ }^{1}$
}

We describe three children who developed an osteosarcoma after receiving treatment for acute lymphoblastic leukemia, which included an allogeneic bone marrow transplant (BMT). We discuss the therapeutic options. None of the patients responded to conventional chemotherapy, but one patient given regorafenib showed a temporary response. We conclude that osteosarcoma after BMT has an aggressive course and it is worth further investigating multikinase inhibitors in this setting.

Keywords: secondary osteosarcoma, bone marrow transplant, total body irradiation, regorafenib

\section{Introduction}

$\mathbf{S}$ ECONDARY MALIGNANCIES AFTER treatment for cancer have become a major health issue since therapies have been more successful and patient survival has increased. ${ }^{1,2}$ Osteosarcoma is the most common primary bone cancer in children and adolescents, and one of the most common secondary malignancies after treatment for solid tumors. It has rarely been reported, however, after treatment for hematological neoplasms: in a series of 152 children with primary acute lymphoblastic leukemia (ALL), only $2.6 \%$ developed secondary bone tumors. ${ }^{3}$

Osteosarcoma after bone marrow transplant (BMT) is also very rare, but tends to be locally invasive, and often metastatic; and the reported prognosis is poor. ${ }^{4-14}$

This report describes three children with ALL who underwent BMT and subsequently developed secondary osteosarcoma. The treatment options are discussed.

\section{Case Report}

\section{Case 1}

A 3-year-old girl was diagnosed with preB-ALL and given chemotherapy according to the Associazione Italiana di Emato-Oncologia Pediatrica (AIEOP) 8803 protocol, which consisted in polychemotherapy associated with cranial irradiation (1800 cGy). A bone marrow relapse occurred 2 years after completing the treatment. The patient was then given second-line chemotherapy and underwent BMT from a mismatched unrelated donor after a conditioning regimen that included thiotepa, cyclophosphamide, and 1200 Gy total body irradiation (TBI). Ten years later (at the age of 15), the patient presented with pain in the left hip. A computed to- mography (CT) scan revealed a lesion $12 \times 10 \mathrm{~cm}$ in size in the left hip bone with a marked periosteal reaction. Biopsy pointed to osteosarcoma with a diffuse chondroblastic component. No metastatic lesions were documented. The search for the p53 mutation was negative.

The patient received chemotherapy with methotrexate, cisplatin, doxorubicin, and ifosfamide according to the Italian ISG/OS-1 osteosarcoma protocol. ${ }^{15}$ After six cycles of therapy, a CT scan revealed local and distant (right hip) disease progression. Despite second-line chemotherapy with high-dose ifosfamide and cyclophosphamide/etoposide, further disease progression was documented. The patient was enrolled in a study on radionuclide therapy with samarium. The severe myelosuppression deriving from this therapy ultimately led to the patient's death 15 months after her osteosarcoma was diagnosed.

\section{Case 2}

A 12-year-old girl was diagnosed with common ALL and treated according to the AIEOP-BFM 2009 protocol (highrisk group). She received a BMT from her brother after a conditioning regimen that included thiotepa, cyclophosphamide, and TBI (1200 cGy). Three years after the BMT (at the age of 17), she complained of pain and swelling of the left leg. Magnetic resonance imaging (MRI) revealed a hypointense 15-cm-long lesion extending from the epiphysis to the midproximal diaphyseal region of the left tibia. A biopsy led to a diagnosis of osteosarcoma. No metastases were found.

Chemotherapy was given according to the Italian ISG/OS2 sarcoma protocol, but the patient developed severe tubulopathy after the first cycle (high-dose methotrexate). A new CT scan revealed the appearance of multiple lung lesions.

\footnotetext{
${ }^{1}$ Hematology and Oncology Unit, Department of Women's and Children's Health, University of Padua, Padua, Italy.

${ }^{2}$ Pediatric Hematology and Oncology Unit, Department of Pediatrics, Bolzano Hospital, Bolzano, Italy.

${ }^{3}$ Fondazione Istituto di Ricerca Pediatrica Città della Speranza, Padua, Italy.
} 
Given her low tolerance of chemotherapy, the patient underwent primary tumor resection followed by gemcitabinedocetaxel therapy. Seven months after she had been diagnosed with osteosarcoma, a local relapse with an intracardiac metastasis was documented. The patient died 2 months later. A diagnosis of Li-Fraumeni syndrome was established postmortem.

\section{Case 3}

A 10-month-old male child was diagnosed with ALL and given chemotherapy according to the AIEOP ALL protocol for infants. During the maintenance phase, the patient had an isolated central nervous system relapse. Second-line chemotherapy was followed by allogeneic BMT after a conditioning regimen that included thiotepa, cyclophosphamide, antithymocyte globulin, and TBI (1440 cGy). Twelve years after his BMT (at the age of 15), the patient complained of pain and swelling of the right thigh. MRI and CT scan revealed a $16 \times 7 \mathrm{~cm}$ lesion and multiple pulmonary nodules. The search for the p53 mutation was negative.

A biopsy identified an osteosarcoma and treatment was initiated according to the ISG/OS-2 protocol, but after two cycles of chemotherapy there was evidence of local and distant tumor progression, and also of pulmonary embolism. The patient was given high-dose ifosfamide, but the treatment was stopped due to neurological toxicity (drowsiness and asthenia, grade 2 CTCAE). Then palliative radiotherapy to the primary tumor was administered. In the light of recent publications, ${ }^{16,17}$ compassionate treatment with regorafenib
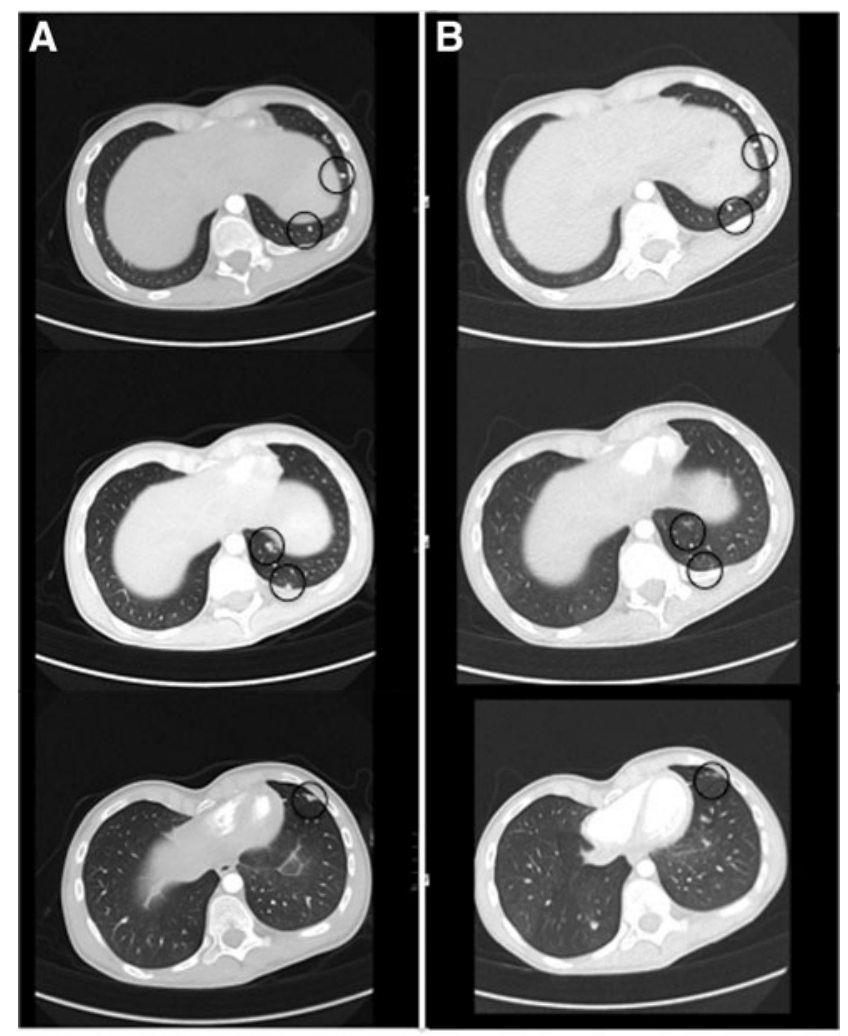

FIG. 1. Lung metastases before treatment with regorafenib (A); partial regression after the first cycle of regorafenib (B). was started at a dose of $100 \mathrm{mg}\left(82 \mathrm{mg} / \mathrm{m}^{2}\right)$ on days $1-21$ of each 28-day cycle. The treatment was well tolerated and, after the first cycle, CT showed a reduction in the number and size of the patient's lung metastases (Fig. 1).

There was also a reduction in the size of the leg mass (to which radiotherapy was administered as well), with a complete regression of the associated pain. On reassessment after the second cycle, an asymptomatic left pneumothorax was identified, which was attributed to cavitation of a subpleural lesion; it was drained surgically. After 4 months of regorafenib, the primary tumor remained stable, but a modest increase in the lung metastases and embolic lesion was documented. The patient died 8 months after starting regorafenib.

\section{Discussion}

A secondary tumor is the most feared consequence of antineoplastic treatment for a primary malignancy in children. Osteosarcoma is a common secondary malignancy, especially in patients previously treated for retinoblastoma, Ewing's sarcoma, ${ }^{18}$ rhabdomyosarcoma, ${ }^{19}$ or Hodgkin lymphoma. ${ }^{20}$ Children are at higher risk if they carry germline mutations predisposing to cancer such as p53 in the Li-Fraumeni syndrome $^{21}$ that we found in one of our patients.

Osteosarcoma after treatment for leukemia is rare. In a population-based cohort study from the Nordic countries, ${ }^{22}$ no osteosarcomas were detected among eight second tumors developing in 895 children with ALL. There were also no bone tumors among 43 second malignancies occurring in a population of 9720 children with ALL in a study performed by the Children's Cancer Study Group. ${ }^{23}$

Second tumors are an important complication of BMT, but secondary osteosarcoma has rarely been reported in this setting. ${ }^{4-14}$ The few reports on the outcome of osteosarcoma after ALL and/or BMT suggest that this is an aggressive tumor with a poor prognosis. We found 21 cases of osteosarcoma secondary to allogeneic BMT in the literature. ${ }^{4-14}$ The median age at diagnosis was 15 years (range 7-26 years). The reason for BMT was ALL in 13 patients, whereas the others were single cases of acute myeloid leukemia, neuroblastoma, chronic granulomatous disease, Blackfan-Diamond anemia, congenital amegakaryocytic thrombocytopenia, myelodysplastic syndrome, and non-Hodgkin lymphoma. Follow-up data were available for 20 patients, and 9 deaths were related to osteosarcoma. Interestingly, a significant proportion of the patients who died had BMT prompted by ALL (7 of 13 patients).

In our experience, osteosarcoma developing after BMT had a very aggressive behavior. All three primary lesions were large, and there were already lung metastases at diagnosis in one case. Our patients were treated with the chemotherapy in use for newly diagnosed osteosarcoma, but response was poor, and further attempts with different regimens failed to improve the situation.

A possible cause of such aggressive behavior is the previous treatment and in particular the administration of TBI. As shown in several studies radio-induced sarcomas ${ }^{24}$ are often resistant to conventional chemotherapy, making highly challenging the management of these diseases, also in the pediatric population. $^{25}$

In our patients chemotherapy tolerance was also poor, with two patients experiencing renal and neurological toxicity. Their previous treatments, including allogeneic BMT, may 
have made these children more susceptible to the negative effects of chemotherapy, even if it was administered 3 and 12 years later.

In this context, the response seen in one patient already after one cycle of regorafenib is of interest, even though the benefit was temporary. The activity of regorafenib (a multikinase inhibitor) against refractory or relapsing osteosarcoma had previously been tested in two studies. Duffaud et al. ${ }^{16}$ treated 38 patients from 18 to 74 years old, 26 given regorafenib, and 12 a placebo. After 8 weeks of treatment, there was no disease progression in $17(65 \%)$ of those given regorafenib (compared with none in a placebo group). The median progression-free survival (PFS) in the regorafenib group was 16.4 weeks (compared with 4.1 weeks with placebo), with a partial response in 2 patients $(8 \%)$ and stable disease in $15(58 \%)$

Similar results were obtained by Davis et al. ${ }^{17}$ in 42 patients from 18 to 76 years (median age 37 years), 22 given regorafenib and 20 a placebo. The median PFS was 3.6 in the regorafenib group and 1.7 months in the placebo group, with a PFS at 16 weeks of $44 \%$ and $10 \%$, respectively $(p=0.027)$. A partial response was observed in three patients treated with regorafenib (13.6\%).

Regorafenib-related toxicity was not negligible. It prompted temporary suspensions of the treatment in $35 \%$ of cases, and dose reductions in $38 \%$ of the cases described by Duffaud. In the article published by Davis et al., ${ }^{17}$ one patient experienced grade 3 pneumothorax, and one had grade 4 colonic perforation, both attributed to regorafenib.

Despite evidence of activity, regorafenib as monotherapy failed to produce any complete response. Its role in the treatment of osteosarcoma needs to be further explored.

In conclusion, our experience confirms that osteosarcoma after BMT is very difficult to treat. It is often resistant to standard chemotherapy, and toxicity is higher than in osteosarcoma patients with no history of BMT. Our experience suggests that regorafenib may have some activity in cases of secondary osteosarcoma.

\section{Acknowledgments}

This study was partially supported by the Alto Adige Peter Pan Association for children with cancer, Bolzano, Italy.

\section{Author Disclosure Statement}

No competing financial interests exist.

\section{Funding Information}

The authors received no specific funding for this work.

\section{References}

1. Choi DK, Helenowski I, Hijiya N. Secondary malignancies in pediatric cancer survivors: perspectives and review of the literature. Int J Cancer. 2014;135(8):1764-73.

2. Tubiana M. Can we reduce the incidence of second primary malignancies occurring after radiotherapy? A critical review. Radiother Oncol. 2009;91:4-15.

3. Meadows A, Friedman D, Neglia J, et al. Second neoplasms in survivors of childhood cancer: findings from the Childhood Cancer Survivor Study cohort. J Clin Oncol. 2009;27: 2356-62.
4. Okada A, Hatori M, Hosaka M, et al. Secondary osteosarcoma arising after treatment for childhood hematologic malignancies. Ups J Med Sci. 2009;114:249-55.

5. Scheuermann A, Phelan R, Browning M. Development of secondary osteosarcoma after TBI and allogeneic bone marrow transplant: a case series of 3 patients. J Pediatr Hematol Oncol. 2020;42:e100-3.

6. Kebudi R, Ozger H, Kizilocak H, et al. Osteosarcoma after hematopoietic stem cell transplantation in children and adolescents: case report and review of the literature. Pediatr Blood Cancer. 2016;63(9):1664-6.

7. Ueki H, Maeda N, Sekimizu M, et al. Osteosarcoma after bone marrow transplantation. J Pediatr Hematol Oncol. 2013;35:134-8.

8. Zils K, Bielack S. Osteosarcoma after bone marrow transplantation: still a challenge. J Pediatr Hematol Oncol. 2015; 37:74.

9. Socié G, Baker KS, Bhatia S. Subsequent malignant neoplasms after hematopoietic cell transplantation. Biol Blood Marrow Transplant. 2012;18:139-50.

10. Shimoda K, Koh K, Uchino S. A case of osteosarcoma after allogeneic bone marrow transplantation for chronic granulomatous disease. J Jpn Pediatr Soc. 2006;111:912.

11. Bielack SS, Rerin JS, Dickerho R, et al. Osteosarcoma after allogenic bone marrow transplantation. A report of four cases from the Cooperative Osteosarcoma Study Group (COSS). Bone Marrow Transplant. 2003;31:3539.

12. Asai T, Myoui A, Fujimoto T, et al. Osteosarcoma after bone marrow transplantation for acute lymphoblastic leukemia. Int J Clin Oncol. 2002;7:318-21.

13. Favre-Schmuziger G, Hofer S, Passweg J, et al. Treatment of solid tumors following allogeneic bone marrow transplantation. Bone Marrow Transplant. 2000;25:895-8.

14. Poustchi-Amin M, Leonidas JC, Elkowitz SS. Simultaneous occurrence of osteosarcoma and osteochondroma following treatment of neuroblastoma with chemotherapy, radiotherapy and bone marrow transplantation. Pediatr Radiol. 1996;26:155-7.

15. Ferrari S, Rufferi P, Cefalo G, et al. Neoadjuvant chemotherapy with methotrexate, cisplatin, and doxorubicin with or without ifosfamide in nonmetastatic osteosarcoma of the extremity: an Italian Sarcoma Group Trial ISG/OS-1. J Clin Oncol. 2012; 30:2112-8.

16. Duffaud F, Mir O, Boudou-Rouquette P, et al. Efficacy and safety of regorafenib in adult patients with metastatic osteosarcoma: a non-comparative, randomised, double-blind, placebo-controlled, phase 2 study. Lancet Oncol. 2019; 20(1):120-33.

17. Davis LE, Bolejack V, Ryan CW, et al. Randomized double-blind phase ii study of regorafenib in patients with metastatic osteosarcoma. J Clin Oncol. 2019;37: 1424-31.

18. Smith LM, Donaldson SS. Incidence and management of secondary malignancies in patients with retinoblastoma and Ewing's sarcoma. Oncology (Williston Park). 1991;5:13541; discussion 142, 147-8.

19. Bechler JR, Robertson WWJ, Meadows AT, Womer RB. Osteosarcoma as a second malignant neoplasm in children. J Bone Joint Surg Am. 1992;74:1079-83.

20. Hawkins MM, Draper GJ, Kingston JE. Incidence of second primary tumours among childhood cancer survivors. $\mathrm{Br}$ J Cancer. 1987;56:339-47.

21. Hameed M, Mendelker D. Tumor syndromes predisponing to osteosarcoma. Adv Anat Pathol. 2018 ; 25(4):217-22. 
22. Nygaard R, Garwicz S, Haldorsen T, et al. Second malignant neoplasms in patients treated for childhood leukemia. A population-based cohort study from the Nordic countries. The Nordic Society of Pediatric Oncology and Hematology (NOPHO). Acta Paediatr Scand. 1991;80: 1220-8.

23. Loning L, Zimmermann M, Reiter A, et al. Secondary neoplasms subsequent to Berlin-Frankfurt-Munster therapy of acute lymphoblastic leukemia in childhood: significantly lower risk without cranial radiotherapy. Blood. 2000;95: 2770-5.

24. Giannini L, Incandela F, Fiore M, et al. Radiation-induced sarcoma of the head and neck: a review of the literature. Front Oncol. 2018;8:449.
25. Koshy M, Paulino AC, Mai WY, Teh BS. Radiationinduced osteosarcomas in the pediatric population. Int $\mathrm{J}$ Radiat Oncol Biol Phys. 200563(4):1169-74.

Address correspondence to: Federico Mercolini, MD Pediatric Hematology and Oncology Unit Department of Pediatrics Bolzano Hospital Bolzano 39100 Italy

Email: federico.mercolini@sabes.it 